\title{
Upper body balance control strategy during continuous 3D postural perturbation in young adults
}

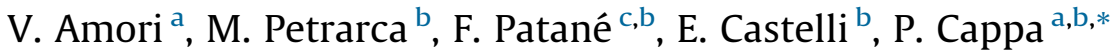 \\ a Department of Mechanical and Aerospace Engineering, “Sapienza” University of Rome, Via Eudossiana, Rome 18 00184, Italy \\ ${ }^{\mathrm{b}}$ Movement Analysis and Robotics Laboratory (MARLab), Neurorehabilitation Division, IRCCS Bambino Gesù Children's Hospital, Via Torre di Palidoro, \\ Passoscuro (Fiumicino), Rome 00050, Italy \\ c “Niccolò Cusano” University, Mechanical Engineering Faculty, Via Don Carlo Gnocchi, Rome 3 00166, Italy
}

\section{A R T I C L E I N F O}

Article history:

Received 29 August 2013

Received in revised form 19 June 2014

Accepted 6 August 2014

\section{Keywords:}

Dynamic posturography

Visual condition

Perturbation frequency

Continuous multiaxial perturbation

\begin{abstract}
A B S T R A C T
We explored how changes in vision and perturbation frequency impacted upright postural control in healthy adults exposed to continuous multiaxial support-surface perturbation. Ten subjects were asked to maintain equilibrium in standing stance with eyes open (EO) and eyes closed (EC) during sinusoidal 3D rotations at $0.25(\mathrm{~L})$ and $0.50 \mathrm{~Hz}(\mathrm{H})$. We measured upper-body kinematics - head, trunk, and pelvis and analyzed differences in horizontal displacements and roll, pitch, and yaw sways. The presence of vision significantly decreased upper-body displacements in the horizontal plane, especially at the head level, while in EC the head was the most unstable segment. $\mathrm{H}$ trials produced a greater segment stabilization compared to L ones in EO and EC. Analysis of sways showed that in EO participants stabilized their posture by reducing the variability of trunk angles; in $\mathrm{H}$ trials a sway decrease for the examined segments was observed in the yaw plane and, for the pelvis only, in the pitch plane. Our results suggest that, during continuous multiaxial perturbations, visual information induced: (i) in L condition, a continuous reconfiguration of multi-body-segments orientation to follow the perturbation; (ii) in $\mathrm{H}$ condition, a compensation for the ongoing perturbation. These findings were not confirmed in EC where the same strategy - that is, the use of the pelvis as a reference frame for the body balance was adopted both in $\mathrm{L}$ and $\mathrm{H}$.
\end{abstract}

(C) 2014 Elsevier B.V. All rights reserved.

\section{Introduction}

Posturography is used to assess balance control deterioration due to age, trauma and disease [1]. It is generally believed that the maintenance of an upright stance during the imposition of an induced external perturbation, that is dynamic posturography, is one of the more complex equilibrium tasks for the Central Nervous System (CNS) to manage [2]. The usefulness of dynamic posturography was also explored from a rehabilitative perspective $[3,4]$.

\footnotetext{
* Corresponding author at: Department of Mechanical and Aerospace Engineering, Sapienza University of Rome, Via Eudossiana, Rome 18 00184, Italy. Tel.: +3906 44585273 .

E-mail addresses: valentinaamori@virgilio.it (V. Amori),

maurizio.petrarca@opbg.net (M. Petrarca), fabrizio.patane@unicusano.it (F. Patané), enrico.castelli@opbg.net (E. Castelli), paolo.cappa@uniroma1.it (P. Cappa).
}

http://dx.doi.org/10.1016/j.gaitpost.2014.08.003

0966-6362/๔ 2014 Elsevier B.V. All rights reserved.
A common method for inducing standing balance perturbation is to use a motorized platform. In the majority of studies, subjects were perturbed with antero/posterior translations [5-7] or rotations only in the pitch [8,9], roll [10] or yaw [11] angles. Previous investigations have described the effect of experimental conditions on posture stabilization and they can be broadly grouped as: those in which visual cues are present [5-11] and those in which perturbation frequency is varied [5-9,11].

The main limitation inherent in the above-mentioned studies is their reliance on postural responses elicited via uniaxial perturbations. Researchers then administered either a combination of abrupt roll-pitch rotations delivered randomly [12-16] or a sequence of unexpected and continuous perturbations [17]. The main finding was that passive and active synergies are triggered and shaped by the CNS eliciting two directionally specific postural responses: one induced by pitch perturbations and the other by a combination of roll and pitch. It was also demonstrated that visual and vestibular information play a relevant role in the control of trunk and head posture $[7,18,19]$. 
Furthermore, real-life situations typically involve continuous periodical multi-directional perturbations such as those that are self-induced by walking. Actually, the ability to manage roll, pitch and yaw combination is necessary to prevent falls, which are common in populations that manifest immature or compromised motor control [3,20-22]. Thus, it can be hypothesized that a more profound examination of postural response could be reached by imposing 3D continuous periodical perturbations, which are generated by a combination of rotations along the roll-pitchyaw axes. In addition 3D perturbation could represent a potentially innovative approach for the rehabilitative assessment and treatment of posture.

In the present study we decided: (i) to use an in-house developed $3 \mathrm{D}$ robotic device [23-28], which could continuously change the rotation direction and which could be set at different amplitudes and frequencies; and (ii) to conduct quantitative assessments of upper body kinematics. In our first working hypothesis we sought to confirm that the presence of visual information, when healthy adults were subjected to continuous 3D perturbation, would induce a greater upper-body stabilization than that occurring when vision is absent. The second working hypothesis was that different perturbation frequencies and the continuous change in perturbation direction would elicit in healthy adult subjects dissimilar compensation strategies of the upper-body.

\section{Methods}

\subsection{Subjects}

Ten healthy adult subjects (six men and four women, age $23.7 \pm 0.7$ years; height $165 \pm 12 \mathrm{~cm}$, and mass $64 \pm 12 \mathrm{~kg}$ ) volunteered for the present study. Participants met the following inclusion criteria: absence of neurological or musculoskeletal disorders, vestibular diseases, dizziness, long term medications, and bone lesions or joint pathologies of the lower limbs in the year prior to the study; it was also ensured that they had normal vision, with or without glasses. All subjects were naïve to the experimental procedures.

All procedures of the present study were approved by the Research Ethics Board of the "Bambino Gesù" Children's Hospital in Rome.

\subsection{Equipment}

Dynamic posturography was performed using an electrically actuated robotic device, the RotoBit ${ }^{3 \mathrm{D}}$, which permits arbitrary rotations - in terms of roll, pitch and yaw directions - around a fixed point. In brief, RotoBit ${ }^{3 \mathrm{D}}$ [23-28] is characterized by: a workspace of about $\pm 10^{\circ}$ for roll and pitch when yaw is in the range $\pm 15^{\circ}$, a phase delay of $1^{\circ}$, and an amplitude error $\leq 1.5 \%$. The RotoBit ${ }^{3 \mathrm{D}}$ was installed in the middle of a 10 -m walkway of the laboratory. A photograph of the RotoBit ${ }^{3 \mathrm{D}}$ is shown in Fig. 1.

Kinematics was recorded by the VICON system (MX 8camera-workstation, Nexus 1.7 software, $200 \mathrm{~Hz}$, PlugInGait marker set based on the Davis protocol [29]). A total of 13 reflective markers (Fig. 2) were placed on the head (2 anterior and 2 posterior), thorax ( 2 on the upper and lower extremities of the sternum; 1 on the 7 th cervical vertebra; 1 on the 10 th dorsal vertebra; and an asymmetric one on the left side), and pelvis ( 2 on the anterior superior iliac spine and 2 on the posterior iliac spine). We decided to study the kinematics of the upper body and to postpone until the ongoing research phase the analysis of how arm movement contributes to balance recovery [30]. A

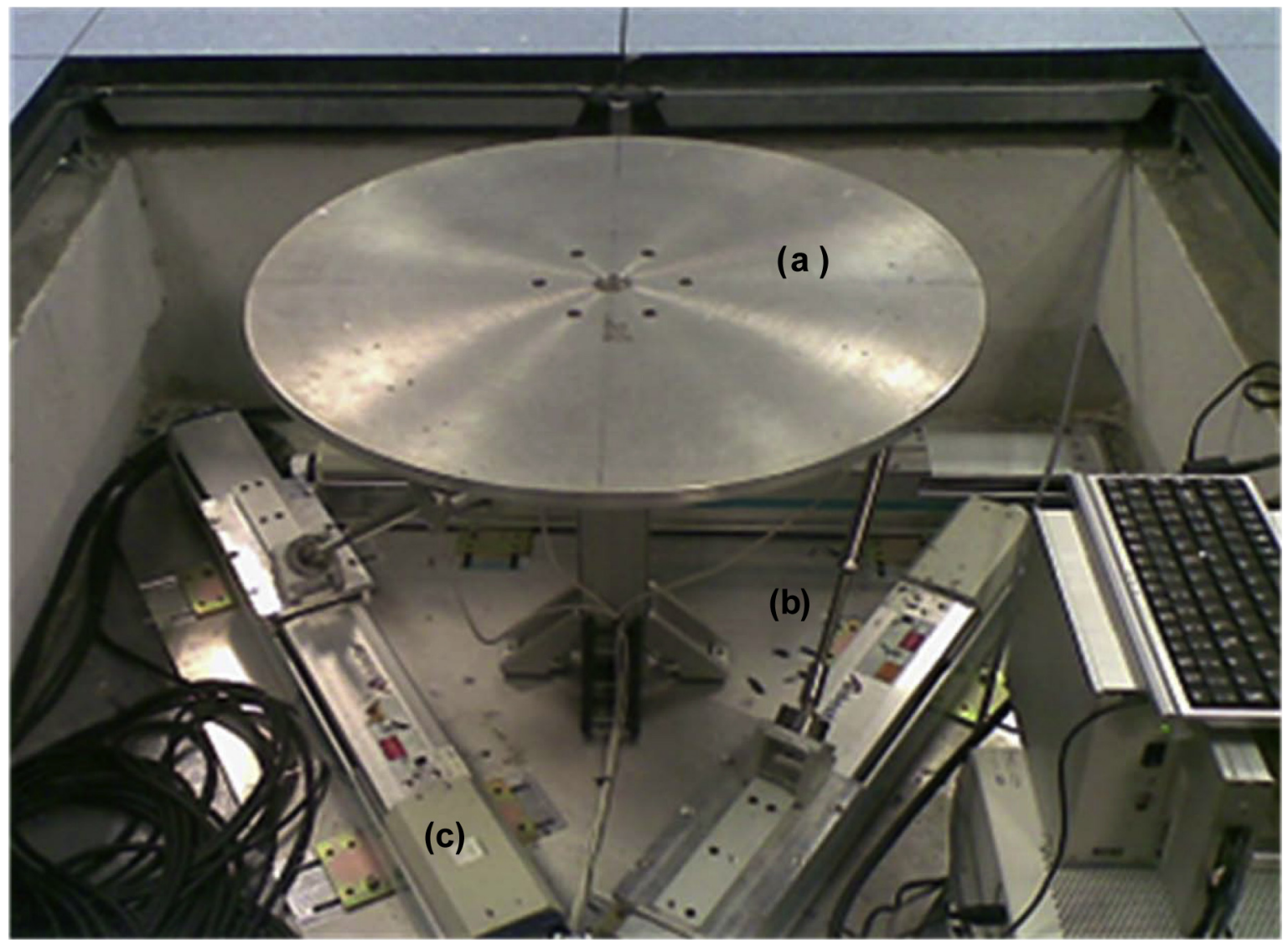

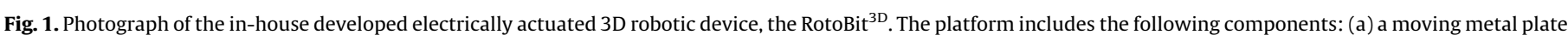

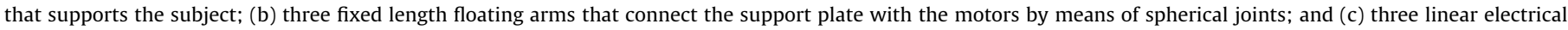
actuators. The robot is concealed in the floor, but in the picture the protective layers have been removed for the viewer's convenience.

Please cite this article in press as: Amori $\mathrm{V}$, et al. Upper body balance control strategy during continuous 3D postural perturbation in young adults. Gait Posture (2014), http://dx.doi.org/10.1016/j.gaitpost.2014.08.003 


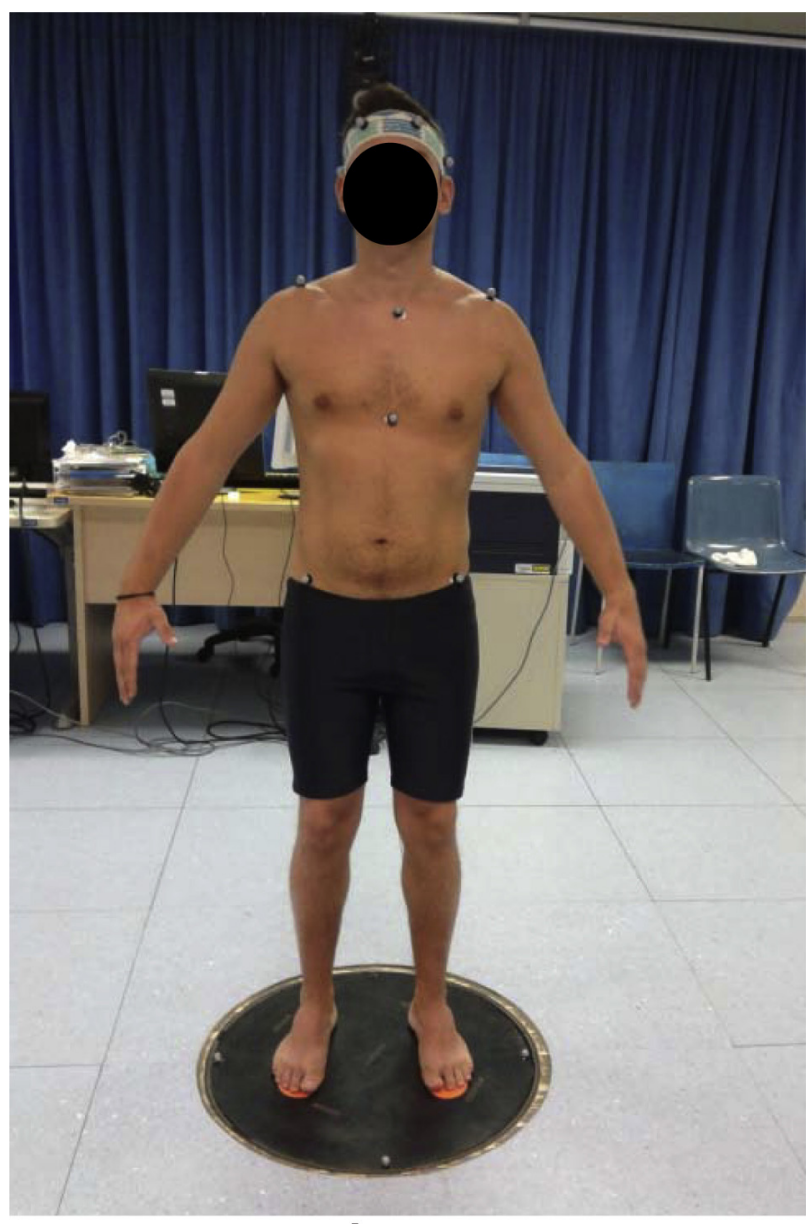

A

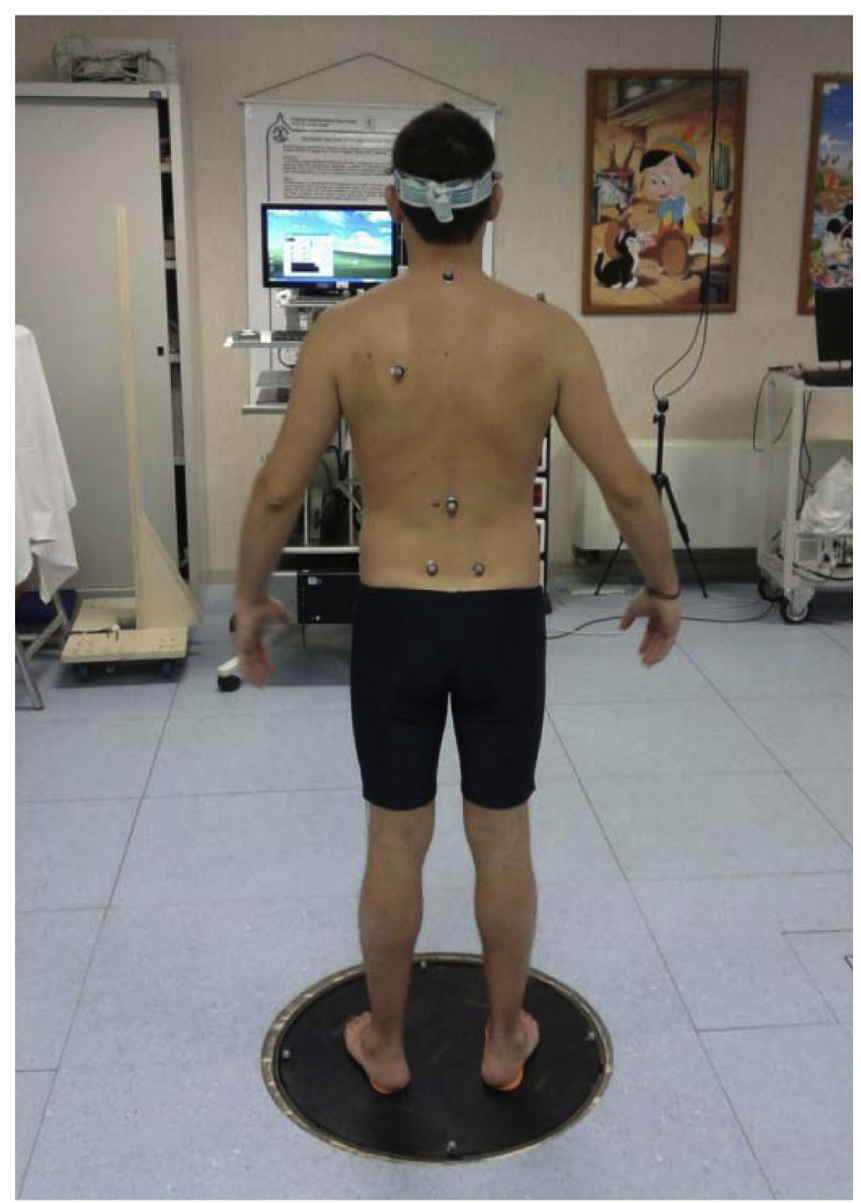

B

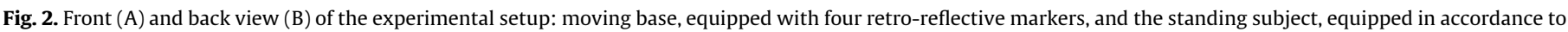
the PlugInGait marker set.

further set of 4 markers was used for recording the platform movements.

\subsection{Procedure}

All subjects wore tight-fitting shorts and female subjects also wore an upper body garment that allowed for the placement of reflective markers. Participants stood barefoot on the platform in their preferred standing position, with arms hanging comfortably at their sides and feet placed symmetrically at the center of rotation of the circular moving base (Fig. 2). Foot position was marked on the platform to assure a consistent position of participants within and across trial blocks. In the eyes open trials (EO), subjects were instructed to look straight ahead and to not gaze at any specific target. In eyes closed trials (EC), the visual feedback was denied to the subjects by having them don eye masks; participants were also instructed to face forward as if looking straight ahead.

Subjects were instructed to maintain equilibrium, restricting their response strategy to a feet-in-place response, unless a fall was imminent; consequently, participants were free to move body segments to compensate for their instability. In order to reduce the risk of falls and to minimize interference from external support, a trainer stayed close to the participant from behind. The trial did not start until the participants indicated they were ready to begin, then a verbal warning was given about five seconds before the platform started moving.
Each trial consisted of a spherical perturbation, lasting $20 \mathrm{~s}$, obtained through the combination of roll-pitch-yaw rotations. The amplitude was set at $6^{\circ}$ for roll and pitch rotations, and $10^{\circ}$ for yaw rotation; the selected combination of rotations was used for every trial and for every subject.

Two frequency levels were selected: low $(\mathrm{L}, 0.25 \mathrm{~Hz})$ and high $(\mathrm{H}, 0.50 \mathrm{~Hz})$. The perturbation frequencies were chosen on the basis of preliminary dry-tests carried out with three healthy adults, who did not participate in the present study. A set of perturbations in the range of $0.2-0.6 \mathrm{~Hz}$ was presented to the subjects. A frequency of $0.25 \mathrm{~Hz}$ was selected because it was the minimum perceived perturbation, while $0.50 \mathrm{~Hz}$ was the highest frequency managed by the participants without stepping or falling. In addition, $\mathrm{L}$ and $\mathrm{H}$ perturbations were easily differentiated by the subjects in both visual conditions. In ongoing research, the selected frequencies will represent the target frequency range for testing and training subjects with postural disorders.

The experimental session consisted of four trial conditions obtained by different combinations of the two visual conditions (EO and EC) and two frequency conditions ( $\mathrm{L}$ and $\mathrm{H}$ ). The four conditions were randomly repeated three times with a timeinterval of at least $30 \mathrm{~s}$ between trials, during which participants were free to move on the still platform in the horizontal pose. The session per subject lasted approximately $20 \mathrm{~min}$.

The initial unpracticed trials for the four conditions were excluded from the sample because they could have produced significantly different reactions from those exhibited during 
Table 1

Mean values \pm SDs of the $95 \%$ confidence ellipse area (CEA) for the head $\left(h_{\mathrm{CEA}}\right)$, trunk $\left(t_{\mathrm{CEA}}\right)$ and pelvis $\left(p_{\mathrm{CEA}}\right)$ in the four experimental conditions.

\begin{tabular}{|c|c|c|c|c|}
\hline \multirow[t]{2}{*}{ Confidence ellipse areas $\left(\mathrm{cm}^{2}\right)$} & \multicolumn{2}{|l|}{ Eyes open (EO) } & \multicolumn{2}{|l|}{ Eyes closed (EC) } \\
\hline & Low frequency (L) $0.25 \mathrm{~Hz}$ & High frequency $(\mathrm{H}) 0.50 \mathrm{~Hz}$ & Low frequency (L) $0.25 \mathrm{~Hz}$ & High frequency $(\mathrm{H}) 0.50 \mathrm{~Hz}$ \\
\hline$h_{\mathrm{CEA}} \mathrm{v} \times \mathrm{f}, \mathrm{v}, \mathrm{f}$ & $80 \pm 36$ & $48 \pm 24$ & $248 \pm 148$ & $165 \pm 71$ \\
\hline$t_{\mathrm{CEA}}$ & $82 \pm 40$ & $48 \pm 26$ & $203 \pm 100$ & $138 \pm 54$ \\
\hline$p_{\text {CEA }}$ & $86 \pm 41$ & $53 \pm 23$ & $138 \pm 46$ & $102 \pm 36$ \\
\hline
\end{tabular}

$\mathrm{v} \times \mathrm{f}$ Significant visual $\times$ frequency interaction effect, $\alpha=0.05$.

${ }^{v}$ Significant visual simple effect or main effect, $\alpha=0.025$ or $\alpha=0.05$, respectively.

${ }^{\mathrm{f}}$ Significant frequency simple effect or main effect, $\alpha=0.025$ or $\alpha=0.05$, respectively.

subsequent trials [31,32]. The unpracticed trials also allowed subjects to familiarize themselves with the equipment and testing procedure prior to the data collection.

\subsection{Data analysis}

To study the upper-body behaviour - i.e., head, trunk and pelvis $(h, t, p)$ - we processed the kinematic data of the segment centers so as to determine their horizontal displacements and sways in terms of roll-pitch-yaw angles. A centered time window of $10 \mathrm{~s}$ in each trial was considered for data analysis to exclude the initial acceleration phase and the final deceleration phase; the transition periods permit the participants to accustom themselves to the perturbation.

We have selected the following indices: (i) the 95\% confidence ellipse areas (CEA) of the trajectories in the horizontal plane [33]; (ii) the absolute sways; and (iii) the relative sways between proximal body-segments.

The confidence ellipse areas - evaluated for head, trunk, and pelvis ( $h_{\text {CEA }}, t_{\text {CEA }}, p_{\text {CEA }}$ ) - were averaged across the four experimental conditions and expressed as means and SDs.

The time course of absolute angles relative to each body segment was categorized into roll-pitch-yaw angles $\left(h_{\mathrm{r}, \mathrm{p}, \mathrm{y}}, t_{\mathrm{r}, \mathrm{p}, \mathrm{y}}\right.$, and $p_{\mathrm{r}, \mathrm{p}, \mathrm{y}}$ ), and after which we calculated the standard deviations $\left(\delta_{-} h_{\mathrm{r}, \mathrm{p}, \mathrm{y}}, \delta_{-} t_{\mathrm{r}, \mathrm{p}, \mathrm{y}}\right.$, and $\left.\delta_{-} p_{\mathrm{r}, \mathrm{p}, \mathrm{y}}\right)$. Considering also the time course of the moving platform $\left(p t_{\mathrm{r}, \mathrm{p}, \mathrm{y}}\right)$, we determined the standard deviations of relative angles: head vs. trunk $\left(\delta \_h / t_{\mathrm{r}, \mathrm{p}, \mathrm{y}}\right)$; trunk vs. pelvis $\left(\delta \_t / p_{\mathrm{r}, \mathrm{p}, \mathrm{y}}\right)$; and pelvis vs. platform $\left(\delta \_p / p t_{\mathrm{r}, \mathrm{p}, \mathrm{y}}\right)$. Absolute and relative sways were normalized to $6^{\circ}$, i.e., the selected amplitude of the platform rotation for roll and pitch axes. Finally, absolute and relative sways were averaged across the four experimental conditions and expressed as means and SDs.

A multifactorial analysis of variance considering interactions was used. Two-way ANOVA was used to study the main effects of visual conditions (2 groups: EO and EC), frequency conditions (2 groups: $\mathrm{L}$ and $\mathrm{H}$ ), and their interactions. The significance level was set at $\alpha=0.05$.

Visual $\times$ frequency interactions, if significant, were further studied, as described in the following. All data were grouped by frequency and a 1-way ANOVA, between EO and EC, was computed for both $\mathrm{L}$ and $\mathrm{H}$, respectively, to study the simple effect of the visual condition. The equivalent procedure was implemented to study the simple effect of the frequency condition for EO and EC, respectively. The significance level was set at $\alpha=0.025$, for the four previously mentioned 1-way ANOVAs, in order to take into account the type I error.

The software package SPSS (IBM-SPSS Inc., USA) was used.

\section{Results}

Our results support the expectation that the visual and frequency conditions influence postural behavior; in fact, a significant visual $\times$ frequency interaction effect and simple effects for both the vision and frequency conditions were observed.

\subsection{Confidence ellipse areas of horizontal displacements}

A significant visual $\times$ frequency interaction $(p=0.039)$, Table 1 , was observed for confidence ellipse areas at the head level, $h_{\text {CEA }}$. The interaction graph (Fig. 3 ) reveals that the interaction was ordinal; the maximum and the minimum values of $h_{\mathrm{CEA}}$ were observed in EC-L and EO-H, respectively. The 1-way ANOVA test conducted on head values showed that there were significant simple effects in both the visual $(p=0.004)$ and frequency $(p=0.003)$ conditions.

\subsection{Absolute and relative sways}

The results for absolute and relative sways, Tables 2 and 3, are clustered in rollpitch-yaw angles.

For the roll angle, a significant main effect of vision emerged only for the trunk $(p=0.002)$ and pelvis sways $(p=0.003)$ and the values were significantly higher in eyes closed condition than in eyes open one. No significant frequency main effect or significant visual $\times$ frequency interaction effect was observed in the roll plane.

For the pitch angle, a significant main effect of the visual condition emerged only on the absolute sway for the trunk ( $p=0.001$ ), the relative sways of head vs. trunk $(p=0.042)$ and trunk vs. pelvis $(p=0.040)$, at levels that were higher in EC than in EO. There was also a significant main effect of frequency on the absolute sway of the pelvis $(p=0.002)$ and relative sway of the pelvis vs. platform $(p=0.000)$. Specifically, absolute sway $\delta \_p$ p assumed higher values in low frequency trials than in high frequency ones, while relative sway $\delta \_p / p t p$ values were lower in L compared with $\mathrm{H}$. No significant visual $\times$ frequency interaction effects were observed in pitch sways.

Finally, in the yaw angles there was a significant main effect of the visual condition on the absolute sway for the head $(p=0.001)$, trunk $(p=0.048)$, and pelvis $(p=0.008)$; in particular, higher values of absolute sways were observed in the EC condition than in the EO condition. Focusing on the absolute sway values, significant main effect of the frequency condition was observed for the head $(p=0.032)$, trunk $(p=0.033)$ and pelvis $(p=0.007), \delta \_t_{\mathrm{y}}$. As regards the relative sways, a significant main effect of the frequency condition was observed for trunk vs. pelvis $(p=0.004)$ and pelvis vs. platform $(p=0.005)$. More specifically, results show that while the previously mentioned absolute sway values were higher in L than in $\mathrm{H}$, the opposite emerged for the relative sway values. No significant visual $\times$ frequency interaction effects were observed for sways in the yaw plane.

While not planned for in the original experimental design, and therefore not supported by an ad-hoc statistical analysis, some further qualitative findings

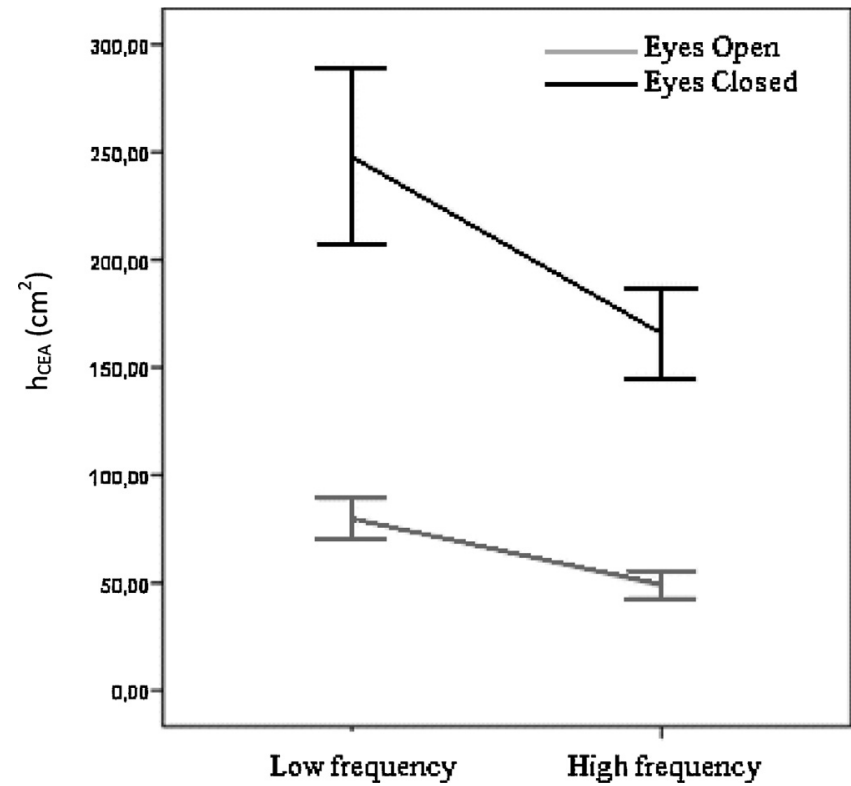

Fig. 3. Confidence ellipse area of the horizontal displacements (CEA) relative to the head: visual $\times$ frequency interaction graph. 
Table 2

Mean values \pm SDs of the absolute sways in the roll, pitch and yaw angles for the head $\left(\delta_{-} h_{\text {r,p.y }}\right)$, trunk $\left(\delta_{-} t_{r, p . y}\right)$ and pelvis $\left(\delta_{-} p_{\text {r,p.y }}\right)$ in the four experimental conditions.

\begin{tabular}{|c|c|c|c|c|}
\hline \multirow[t]{2}{*}{ Absolute sways [\%] } & \multicolumn{2}{|l|}{ Eyes open (EO) } & \multicolumn{2}{|l|}{ Eyes closed (EC) } \\
\hline & Low frequency (L) $\mathrm{Hz}$ & High frequency $(\mathrm{H}) \mathrm{Hz}$ & Low frequency (L) $\mathrm{Hz}$ & High frequency $(\mathrm{H}) \mathrm{Hz}$ \\
\hline \multicolumn{5}{|l|}{ Roll } \\
\hline$\delta \_h_{\mathrm{r}}$ & $47 \pm 30$ & $45 \pm 23$ & $45 \pm 15$ & $50 \pm 17$ \\
\hline$\delta \_t_{\mathrm{r}}^{\mathrm{v}}$ & $29 \pm 7$ & $30 \pm 8$ & $51 \pm 30$ & $51 \pm 24$ \\
\hline$\delta \_p_{\mathrm{r}}^{\mathrm{v}}$ & $34 \pm 9$ & $33 \pm 8$ & $46 \pm 21$ & $41 \pm 15$ \\
\hline \multicolumn{5}{|l|}{ Pitch } \\
\hline$\delta \_h_{\mathrm{p}}$ & $39 \pm 18$ & $42 \pm 22$ & $36 \pm 14$ & $37 \pm 20$ \\
\hline$\delta \_t_{\mathrm{p}}^{\mathrm{v}}$ & $27 \pm 7$ & $27 \pm 8$ & $40 \pm 15$ & $35 \pm 10$ \\
\hline$\delta \_p_{\mathrm{p}}{ }^{\mathrm{f}}$ & $46 \pm 15$ & $39 \pm 12$ & $48 \pm 17$ & $42 \pm 11$ \\
\hline \multicolumn{5}{|l|}{ Yaw } \\
\hline$\delta \_h_{\mathrm{y}}^{\mathrm{v}, \mathrm{f}}$ & $75 \pm 28$ & $68 \pm 34$ & $90 \pm 35$ & $76 \pm 33$ \\
\hline$\delta \_t_{\mathrm{y}}^{\mathrm{v}, \mathrm{f}}$ & $91 \pm 21$ & $87 \pm 29$ & $101 \pm 31$ & $91 \pm 29$ \\
\hline$\delta \_p_{\mathrm{y}} \mathrm{v}, \mathrm{f}$ & $92 \pm 19$ & $84 \pm 25$ & $101 \pm 24$ & $91 \pm 22$ \\
\hline
\end{tabular}

$\mathrm{v} \times \mathrm{f}$ Significant visual $\times$ frequency interaction effect, $\alpha=0.05$.

v Significant visual simple effect or main effect, $\alpha=0.025$ or $\alpha=0.05$, respectively.

f Significant frequency simple effect or main effect, $\alpha=0.025$ or $\alpha=0.05$, respectively.

emerged from a comparative examination of the body segment data in the same trial condition.

When the visual cue was denied and the moving platform was set to $0.25 \mathrm{~Hz}$, the means of CEA's were generally higher at the head than at the trunk and pelvis, and higher at the trunk than pelvis. In the same visual condition and by increasing the perturbation frequency to $0.50 \mathrm{~Hz}$, the mean value of CEA at the head level was higher than that of trunk, and that at the trunk was higher than that of pelvis.

Concerning the roll angles, mean values of the absolute sway for the head were generally higher than those achieved by the trunk only in EO, whereas in EC they were lower or comparable. In EO, the trunk exhibited lower sways compared not only to the head, but also to the pelvis, whereas this trend was not observed in the EC.

Furthermore, irrespective of the visual condition, as regards the yaw angle, mean values of the absolute sway for the head were lower than those of trunk and the pelvis, while SDs increased going from the pelvis to the head.

\section{Discussion}

Our findings highlighted that healthy adult subjects respond differently to the administration of continuous 3D support-surface motion when the visual information and frequency perturbation are changed. The visual cues drive the selection of the body segment used as a reference frame: the head when vision is allowed, the pelvis when vision is denied. Low values of oscillation frequency induce a balance control aimed at following the perturbation, while higher frequencies elicit a behavior aimed at compensating for the perturbation. Hence, the previously mentioned findings, together with the observed postural flexibility, support the hypothesis that $3 \mathrm{D}$ perturbations induce healthy subjects to enrich their perception of the ongoing postural configuration and to re-weight the relative contribution of body-segment activity. Finally, the previously indicated outcomes represent a rationale for the proposal of personalized goal-driven learning and rehabilitative processes.

\subsection{Confidence ellipse areas of horizontal displacements}

Subjects exhibited higher values of CEA's in eyes closed EC trials than in eyes open EO ones, especially at the head level, confirming the findings of previous studies [5-7] obtained with continuous anterior/posterior translating perturbations. In fact, in our study healthy subjects: (i) use the incoming information to select a feedforward mechanism to obtain upper-body stabilization; and (ii) reweight multi-sensory information as evidenced by the differences in the results obtained in the EO trial, where visual information is dominant, with respect to the EC trials, where vestibular and proprioceptive information are prevalent.

Concerning the effect of perturbation frequency, CEA's were higher in trials carried out at low frequency condition, L, than in high frequency one, $\mathrm{H}$. The observed results suggest that the $\mathrm{L}$ condition could be easily tolerated by the subjects who permit the perturbation to dislocate body segments in space. In trials conducted with high perturbation frequency a more complex behavior emerged; in fact, the significant reduction of CEA's indicates a strategy modification to achieve postural balance and participants preferred to compensate for the imposed perturbations. In previous

Table 3

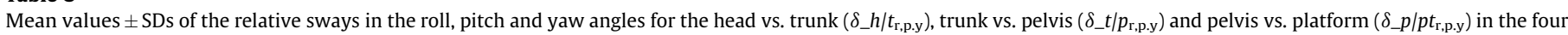
experimental conditions.

\begin{tabular}{|c|c|c|c|c|}
\hline \multirow[t]{2}{*}{ Relative sways [\%] } & \multicolumn{2}{|l|}{ Eyes open (EO) } & \multicolumn{2}{|l|}{ Eyes closed (EC) } \\
\hline & Low frequency $(\mathrm{L}) \mathrm{Hz}$ & High frequency $(\mathrm{H}) \mathrm{Hz}$ & Low frequency $(\mathrm{L}) \mathrm{Hz}$ & High frequency $(\mathrm{H}) \mathrm{Hz}$ \\
\hline \multicolumn{5}{|l|}{ Roll } \\
\hline$\delta \_h / t_{\mathrm{r}}$ & $35 \pm 23$ & $39 \pm 25$ & $34 \pm 14$ & $37 \pm 17$ \\
\hline$\delta \_t / p_{\mathrm{r}}$ & $54 \pm 18$ & $49 \pm 14$ & $49 \pm 18$ & $47 \pm 18$ \\
\hline$\delta \_p / p t_{\mathrm{r}}$ & $115 \pm 11$ & $116 \pm 9$ & $113 \pm 19$ & $113 \pm 14$ \\
\hline \multicolumn{5}{|l|}{ Pitch } \\
\hline$\delta \_h / t_{\mathrm{p}}{ }^{\mathrm{v}}$ & $48 \pm 31$ & $42 \pm 19$ & $54 \pm 25$ & $53 \pm 22$ \\
\hline$\delta_{-} t / p_{\mathrm{p}}{ }^{\mathrm{v}}$ & $34 \pm 14$ & $37 \pm 12$ & $38 \pm 15$ & $41 \pm 23$ \\
\hline$\delta \_p / p t_{\mathrm{p}}{ }^{\mathrm{f}}$ & $71 \pm 23$ & $83 \pm 22$ & $69 \pm 24$ & $80 \pm 19$ \\
\hline \multicolumn{5}{|l|}{ Yaw } \\
\hline$\delta \_h / t_{\mathrm{y}}$ & $36 \pm 22$ & $44 \pm 23$ & $35 \pm 23$ & $40 \pm 17$ \\
\hline$\delta \_t / p_{\mathrm{y}}{ }^{\mathrm{f}}$ & $19 \pm 5$ & $24 \pm 8$ & $20 \pm 6$ & $25 \pm 58$ \\
\hline$\delta \_p / p t_{\mathrm{y}}{ }^{\mathrm{f}}$ & $51 \pm 15$ & $68 \pm 16$ & $53 \pm 17$ & $59 \pm 17$ \\
\hline
\end{tabular}

$\mathrm{v} \times \mathrm{f}$ Significant visual $\times$ frequency interaction effect, $\alpha=0.05$.

v Significant visual simple effect or main effect, $\alpha=0.025$ or $\alpha=0.05$, respectively.

${ }^{f}$ Significant frequency simple effect or main effect, $\alpha=0.025$ or $\alpha=0.05$, respectively. 
studies, the effect of frequency in stabilizing posture has been assessed by inducing visually simulated motion in differently structured environments, during which subjects dissociate visual and body sensorial information, centering the spatial reference on body segments [34]. Conversely, when mechanical perturbations are applied, the spatial reference frame is centered on the visual cue. In that case it was demonstrated that the exposure of healthy subjects to a moving visual environment could induce a rich variety of patterns which are dependent on the driving frequency [35]. In particular, slow visual field oscillation $(\approx 0.2-0.3 \mathrm{~Hz})$ almost exclusively induced absolute coordination, i.e., stimulus movement and postural response were phase-locked, while faster visual scene oscillation $(\geq 0.4 \mathrm{~Hz})$ may or may not have induced coherent postural sway. Thus, their results suggest that vision was prominent at slow frequency, while at high frequency body mechanical properties are also involved in balance control; the variety of behaviors could be explained by subject dexterity based on previous experiences. The experimental paradigm based on moving the support base showed a greater effect of visual feedback in the body stabilization. Indeed, by imposing a continuous anterior/posterior translating perturbation, Buchanan et al. [5], Corna et al. [6] and Berger et al. [36] observed that, for frequencies $\geq 0.5 \mathrm{~Hz}$, subjects preferred to stabilize the posture with a greater damping of head and trunk, always in the anterior/posterior direction. The body damping observed in our experiment carried out at $0.5 \mathrm{~Hz}$ is then in accord with the findings of previous studies.

As regards the visual $\times$ frequency interaction effects, the greater increase in head displacement observed between EC-L and EC-H with respect to the increase observed between EO-L and EO-H could be interpreted as a behavior specifically selected by participants. They preferred, due to a longer latency of the response from vestibular input compared to visual input, to increase the signals provided by the vestibular system, as described by Schieppati et al. [7]. The observed higher head displacement also determines an increased response of the receptors positioned under the feet due to the relevant contribution of the head in COP displacements. In addition, while in the eyes open condition the more perturbed segment is the pelvis, in the eyes closed condition it is the less perturbed segment, which implies that in the absence of vision, the pelvis is used as a reference frame to achieve body balance.

\subsection{Absolute and relative sways}

Our results show that the trunk increased the roll sway in eyes closed EC trials, whereas the visual cues did not affect the head roll and pitch sways. These results show that in EC participants did not reduce the head sways as demonstrated also by the increase of $h_{\mathrm{CEA}}$; while results on yaw angles confirm the role of visual information in compensating the upcoming perturbation [11]. Our findings highlight the role of vision in upper-body stabilization, achieved via an active rearrangement of body-segment configuration to compensate for the ongoing perturbation. Moreover, in the EC condition the reduction of the absolute pitch sway of the pelvis when perturbation with a higher frequency is imposed, the contemporaneous highest value of relative sway of the pelvis vs. platform observed in roll-pitch-yaw angles, together with the above mentioned CEA data, confirmed the hypothesis that when vision is denied the pelvis is used as a reference frame for the body to finalize balance recovery. This finding is also supported in [37], where translation frequencies $<0.6 \mathrm{~Hz}$ and $\geq 0.6 \mathrm{~Hz}$ involved the ankle and hip strategy, respectively.

Furthermore, in eyes open EO trials, participants responded differently in the roll angle with respect to the pitch and yaw angles. Concerning the roll angle, sway was lower for the neck than for the lumbar joint, while the opposite was observed for pitch and yaw planes. In EO trials absolute roll sway was higher for the head than for the trunk; similar behavior was observed in the pitch but not in the yaw plane. The distinctive roll behavior was also observed in previous works where uniaxial perturbation was used $[12,13]$. One explanation could be that complex perturbations are compensated for by merging two main strategies: (i) by roll rotations based on hip ab/adduction; and (ii) by pitch rotations based on hip flexion-extension. It could be hypothesized that these behaviors are driven by body constraints resulting from the chosen standing position (i.e., with parallel feet) and instruction given to maintain equilibrium which determines a higher instability in the anterior/posterior than in the medio/lateral direction. Moreover, the high frequency $\mathrm{H}$ condition globally reduced the yaw angles of the upper-body, even though a significant difference appeared only for head and pelvis, forcing all the relative sways to increase. These findings suggest that the inter-joint flexibility was predominantly used in $\mathrm{H}$ to compensate for the perturbation at the pelvis and head levels. As in the findings reported in [11], our subjects chose to selectively release the degrees of freedom of the body rather than counteract the dynamic input by increasing joint stiffness.

\section{Acknowledgments}

This project was supported by two research grants from the Italian Health Ministry (Grant 'Pilot study on a novel typology of medical devices: robotic systems for rehabilitation and telerehabilitation'; PI: Paolo Cappa) and the Italian Ministry of University (PRIN 2006 grant 'Development of novel methods for the measurement of mechanical variables to optimize the movement rehabilitation'; PI: Paolo Cappa).

\section{Conflict of interest statement}

None of the authors of this manuscript has any financial or personal relationship with other people or organization that could inappropriately influence their work.

\section{References}

[1] Visser JE, Carpenter MG, van der Kooij H, Bloem BR. The clinical utility of posturography. Clin Neurophysiol 2008;119:2424-36.

[2] Vrieling AH, van Keekenb HG, Schoppenc T, Ottenb E, Hof AL, Halbertsma JPK, et al. Balance control on a moving platform in unilateral lower limb amputees. Gait Post 2008;28(2):222-8

[3] Petrarca M, Cappa P, Zanelli G, Armando M, Castelli E, Berthoz A. Spatial rotational orientation ability in standing children with cerebral palsy. Gait Post 2013;37(4):494-9.

[4] Nardone A, Godi M, Artuso A, Schieppati M. Balance rehabilitation by moving platform and exercises in patients with neuropathy or vestibular deficit. Arch Phys Med Rehabil 2010;91(12):1869-77.

[5] Buchanan JJ, Horak FB. Emergence of postural patterns as a function of vision and translation frequency. J Neurophysiol 1999;81(5):2325-39.

[6] Corna S, Tarantola J, Nardone A, Giordano A, Schieppati M. Standing on a continuously moving platform: is body inertia counteracted or exploited? Exp Brain Res 1999;124(3):331-41.

[7] Schieppati M, Giordano A, Nardone A. Variability in a dynamic postural task attests ample flexibility in balance control mechanisms. Exp Brain Res 2002;144(2):200-10.

[8] Gurfinkel VS, Lipshits MI, Mori S, Popov KE. Postural reactions to the controlled sinusoidal displacement of the supporting platform. Agressologie 1976;17:71-6.

[9] Walsh EG. Standing man, slow rhythmic tilting, importance of vision. Agressologie 1973;14(C):79-85.

[10] Pozzo T, Levik Y, Berthoz A. Head and trunk movements in the frontal plane during complex dynamic equilibrium tasks in humans. Exp Brain Res 1995;106(2):327-38

[11] Cappa P, Patanè F, Rossi S, Petrarca M, Castelli E, Berthoz A. Effect of changing visual condition and frequency of horizontal oscillations on postural balance of standing healthy subjects. Gait Post 2008;28(4):615-26.

[12] Carpenter MG, Allum JH, Honegger F. Directional sensitivity of stretch reflexes and balance corrections for normal subjects in the roll and pitch planes. Exp Brain Res 1999;129(1):93-113.

[13] Carpenter MG, Allum JH, Honegger F. Vestibular influences on human postural control in combinations of pitch and roll planes reveal differences in spatiotemporal processing. Exp Brain Res 2001;140(1):95-111.

[14] Allum JH, Carpenter MG, Honegger F, Adkin AL, Bloem BR. Age-dependent variations in the directional sensitivity of balance corrections and compensatory arm movements in man. J Physiol 2002;542(Pt 2):643-63. 
G Mode

GAIPOS-4278; No. of Pages 7

V. Amor et al./Gait E Posture $x x x(2014) x x x-x x x$

7

[15] Gruneberg C, Duysens J, Honegger F, Allium JH. Spatiotemporal separation of roll and pitch balance-correcting commands in humans. J Neurophysiol 2005;94(5):3143-58.

[16] Jung UM, Horlings CG, Honegger F, Duysens JE, Allium JH. Control of roll and pitch motion during multidirectional balance perturbations. Exp Brain Res 2009;194(4):631-45.

[17] Tai YC, Hsieh LF, Yang S. Age-related changes in posture response under a continuous and unexpected perturbation. J Biomech 2014;47(2):482-90.

[18] Keshner EA, Woollacott MH, Debu B. Neck, trunk and limb muscle responses during postural perturbations in humans. Exp Brain Res $1988 ; 71: 455-66$.

[19] Allium JH, Bloem BR, Carpenter MG, Honegger F. Differential diagnosis of proprioceptive and vestibular deficits using dynamic support-surface posterography. Gait Post 2001;14(3):217-26.

[20] Thigpen MT, Light KE. turning difficulty characteristics of adults aged 65 years or older. Phys There 2000;80(12):1174-87.

[21] Buderath P, Gärtner K, Firings M, Christiansen H, Schoch B, Konczak J, et al. Postural and gait performance in children with attention deficit/hyperactivity disorder. Gait Post 2009;29:249-54.

[22] Finding O, Sellner J, Meier N, Plum JH, Vibert D, Lienert C, et al. Trunk sway in mildly disabled multiple sclerosis patients with and without balance inpairment. Exp Brain Res 2011;213(4):363-70.

[23] Cappa P, Patané F. Motorized platform for the therapeutic treatment of patients. In: European Patent Apple. EP1880702A2; 2006.

[24] Rossi SP, Patané F, Scalise F, Marchionni L, Cuppa P. Centre of pressure in dynamic posturography: a comparison among systems based on a pressure matrix and a force platform. Measure Sci Technol 2010;21(1).

[25] Cappa P, Jackson JL, Patané F. Moment measurement accuracy of a parallel spherical robot for dynamic posturography. IEEE Trans Boomed Eng 2010;57(5):1198-208.

[26] Patané F, Cuppa P. A 3-D of parallel robot with a spherical motion for the rehabilitation and evaluation of balance performance. IEEE Trans Neural Syst Rehabil Eng 2010;19(2):157-66.
[27] Patané F, Ross S, Cuppa P. Experimental validation of an impedance controlled parallel robot for postural rehabilitation. In: Proceedings of the th IEEE RAS/ EMBS International Conference on Biomedical Robotics and Biomechatronics; 2012. p. 1077-80.

[28] De Marchis C, Patané F, Petrarch M, Carnies S, Schmid M, Conforto S, et al. EMG and kinematics assessment of postural responses during balance perturbation on a Bd robotic platform: preliminary results in children with hemiplegia. In: IFMBE Proceedings of the XIII Mediterranean Conference on Medical and Biological Engineering and Computing 2013, vol. 41; 2014. p. 69-72.

[29] Davis RB, Õunpuu S, Tybursk iD, Gage JR. A gait analysis data collection and reduction technique. Hum Move Sci 1991;10:575-87.

[30] Pijnappels M, Kingma I, Wezenberg D, Reurink G, van Dieën JH. Armed against falls: the contribution of arm movements to balance recovery after tripping. Exp Brain Res 2010;201:689-99.

[31] Bloem BR, van Vugt JP, Beckley DJ, Remler MP, kos RA. Habituation of lower leg stretch responses in Parkinson's disease. Electroencephalogr Chin Nerophysio 1998;109(1):73-7.

[32] Visser JE, Dude Nijhuis LB, Jansen L, Bastiaanse CM, Berm GF, Duysens J, et al. Dynamic posturography in Parkinson's disease: diagnostic utility of the first trial effect. Neuroscience 2010;168(2):387-94.

[33] Sokal RR, Rohlf FJ. Biometry. 3rd ed. New York: W.H. Freeman and Company; 1995.

[34] van Asten WN, Gielen CC, Denier van der Con JJ. Postural adjustments induced by simulated motion of differently structured environments. Exp Brain Res 1988;73(2):371-83.

[35] Dijkstra TM, Schöner G, Giese MA, Gielen CC. Frequency dependence of the action-perception cycle for postural control in a moving visual environment: relative phase dynamics. Biol Cybern 1994;71(6):489-501.

[36] Berger W, Tripped M, Assaiante C, Zijlstra W, Dietz V. Developmental aspects of equilibrium control during stance: a kinematic and EMG study. Gait Post 1995;3(3):149-55.

[37] Buchanan JJ, Horak FB. Voluntary control of postural equilibrium patterns. Behav Brain Res 2003;143(2):121-40.

Please cite this article in press as: Amori V, et al. Upper body balance control strategy during continuous 3D postural perturbation in young adults. Gait Posture (2014), http://dx.doi.org/10.1016/j.gaitpost.2014.08.003 